\title{
Mn2+ complex-modified polydopamine- and dual emissive carbon dots based nanoparticles for in vitro and in vivo trimodality fluorescent, photothermal, and magnetic resonance imaging
}

Zhang, Ming; Zheng, Tao; Sheng, Bulei; Wu, Fan; Zhang, Qicheng; Wang, Wengtao; Shen, Jian; Zhou, Ninglin; Sun, Yi

Published in:

Chemical Engineering Journal

Link to article, DOI:

10.1016/j.cej.2019.05.107

Publication date:

2019

Document Version

Peer reviewed version

Link back to DTU Orbit

Citation (APA):

Zhang, M., Zheng, T., Sheng, B., Wu, F., Zhang, Q., Wang, W., Shen, J., Zhou, N., \& Sun, Y. (2019). Mn ${ }^{2+}$ complex-modified polydopamine- and dual emissive carbon dots based nanoparticles for in vitro and in vivo trimodality fluorescent, photothermal, and magnetic resonance imaging. Chemical Engineering Journal, 373 , 1054-1063. https://doi.org/10.1016/j.cej.2019.05.107

\section{General rights}

Copyright and moral rights for the publications made accessible in the public portal are retained by the authors and/or other copyright owners and it is a condition of accessing publications that users recognise and abide by the legal requirements associated with these rights.

- Users may download and print one copy of any publication from the public portal for the purpose of private study or research.

- You may not further distribute the material or use it for any profit-making activity or commercial gain

- You may freely distribute the URL identifying the publication in the public portal 


\title{
$\mathrm{Mn}^{2+}$ Complex-Modified Polydopamine- and Dual Emissive
}

\author{
Carbon dots Based Nanoparticles for in Vitro and in Vivo
}

Trimodality Fluorescent, Photothermal, and Magnetic

\section{Resonance Imaging}

Ming Zhang, ${ }^{a, b}$ Tao Zheng, ${ }^{a}$ Bulei Sheng, ${ }^{c}$ Fan Wu, ${ }^{b}$ Qicheng Zhang, ${ }^{b}$ Wentao Wang, ${ }^{b}$ Jian Shen, ${ }^{b}$ Ninglin Zhou, ${ }^{b^{*}}$ Yi Sun ${ }^{{ }^{*}}$

${ }^{a}$ Department of Health Technology, Technical University of Denmark, Kongens Lyngby DK-2800, Denmark.

bJiangsu Collaborative Innovation Center for Biomedical Functional Materials, School of Chemistry and Materials Science, Nanjing Normal University, Nanjing 210023, P. R. China.

${ }^{c}$ Department of Food Science, Aarhus University, 8830 Tjele, Denmark

Corresponding author: Ninglin Zhou, zhouninglin@njnu.edu.cn; SunYi,suyi@dtu.dk

\begin{abstract}
Multimodality molecular imaging has attracted more and more attention recently, as it possesses novel multiple imaging patterns via combining information from several independent molecular imaging techniques. Here, we investigated the multifunctional $\mathrm{Mn}^{2+}$ complex-modified polydopamine (PDA) and dual emissive carbon dots-based nanoparticles (NPs) for trimodality fluorescent, photothermal and magnetic resonance (MR) imaging in vitro and in vivo. In our system, N-doped carbon dots (N-CDs), a kind of green and red emissive CDs, were loaded or embedded onto the PDA NPs for fluorescent imaging. PDA NPs acted as a photothermal agent which showed obvious near-infrared (NIR) absorbance and high photothermal conversion efficiency (38.2\%). Moreover, due to the automatic chelation of $\mathrm{Mn}^{2+}$ ions, PDA@N-CDs(Mn) NPs could offer contrast for magnetic resonance (MR) imaging. The application of PDA@N-CDs(Mn) NPs for fluorescent, photothermal, and MR in vivo imaging could were successfully be demonstrated successfully. Besides, the the cytotoxicity analysis, hemolytic test, histological analysis of viscera sections, and as well as blood biochemical analysis have showed that the PDA@N-CDs(Mn) NPs had
\end{abstract}


excellent biocompatibility and low biological toxicity.

Keywords: polydopamine, photothermal, carbon dots, fluorescent, $\mathrm{Mn}^{2+}$ complex, magnetic resonance imaging

\section{Introduction}

Molecular imaging techniques, such as fluorescence, photothermal, and magnetic resonance (MR) imaging, play an important role in medicine and biomedical research. However, information obtained from single modal molecular imaging is unsatisfactory for in vivo performance [1-3]. For example, fluorescence and photothermal imaging with high sensitivity is often restricted because of the limited penetration depth, whereas MR imaging with a high spatial resolution are of insufficient sensitivity $[4,5]$. To remove these intrinsic restrictions, there is an emerging need to gather multiple modal imaging techniques. Therefore, the combination of fluorescent, photothermal, and MR imaging into one single strategy can be advantageous for tumor detection, as it provides deeper tissue penetration and maintains imaging sensitivity compared to other single optical imaging methods $[6,7]$. As far as we know, nano-diagnostics has greatly promoted the development of the contrast agent for multimodal molecular imaging, because of its ability to integrate multiple functions to a single nanoparticle (NPs) $[8,9]$. Therefore, current researches are going forward in the study of low cytotoxic and high photostable nanomaterials for better imaging.

At present, carbon dots (CDs) is a new face type of carbon nanomaterials, which have the outstanding properties of semiconductor quantum dots (SQDs) but with no intrinsic toxicity [10-13]. Besides, CDs have been proved as efficient catalysts and are commonly used in energy devices by exploring their fluorescent and photochemical properties [14-16]. CDs can give fluorescence emission at multiple wavelengths, but the tunability was difficult to achieve, which limited their broad applications in bioimaging $[17,18]$. Excitation independent CDs have been reported, but they only restricted to blue to green CDs only [19]. Therefore, it is still hard to obtain tunable 
CDs with a broad range of green to red fluorescence emissions. Moreover, the performance of CDs synthesized by current methods were subjected to some limitations $[20,21]$. As a result, developing a new method to prepare green and red dual emission CDs would greatly facilitate the use of CDs as a fluorescence imaging agent for cancer diagnosis.

Recently, increasing attention has been paid on polydopamine (PDA) NPs, because of their good biocompatibility and intense near-infrared (NIR) absorption [22, 23]. PDA has similar structure as naturally existing melanin, and they can be prepared from dopamine with an ordinary and effective method. Moreover, their surface can be easily modified with thiol- and amino-terminated molecules through Schiff base reaction or Michael addition, which enables the loading of other attractive molecules [24-26]. Therefore, PDA NPs is a good candidate for clinical photothermal imaging. In addition, abundant functional groups (e.g. catechol, carboxyl, and amino) in PDA also endowed their capability indirectly to chelate different metal ions [25]. For instance, there was one study demonstrated that $\mathrm{Fe}^{3+}, \mathrm{Mn}^{2+}$, or $\mathrm{Gd}^{3+}$ ions could devote PDA-based nanomaterials as MR imaging contrast agents [27].

In the present study, PDA with good biocompatibility was employed as a coating for hydrophobic nanocrystals, so that multi-core products of the PDA@N-CDs NPs were obtained. Then, $\mathrm{Mn}^{2+}$, a $\mathrm{MR}$ imaging contrast agent, was integrated with phenolic hydroxyl groups of PDA@N-CDs NPs via chelation. Their morphology, optical, phtotheraml, and magnetic properties were investigated. Fluorescent imaging for living cervix cancer (HeLa) and pulmonary adenocarcinoma (A549) cells was studied with the PDA@N-CDs(Mn) NPs. Furthermore, PDA@N-CDs(Mn) NPs were applied in the trimodal fluorescent, photothermal, and MR imaging for small animals, both in vivo and ex vivo. In addition, the toxicological responses of PDA@N-CDs(Mn) NPs was evaluated by the histological analyses and blood biochemical analysis.

\section{Results and discussion}

\subsection{Characterization of the PDA@N-CDs(Mn) NPs.}




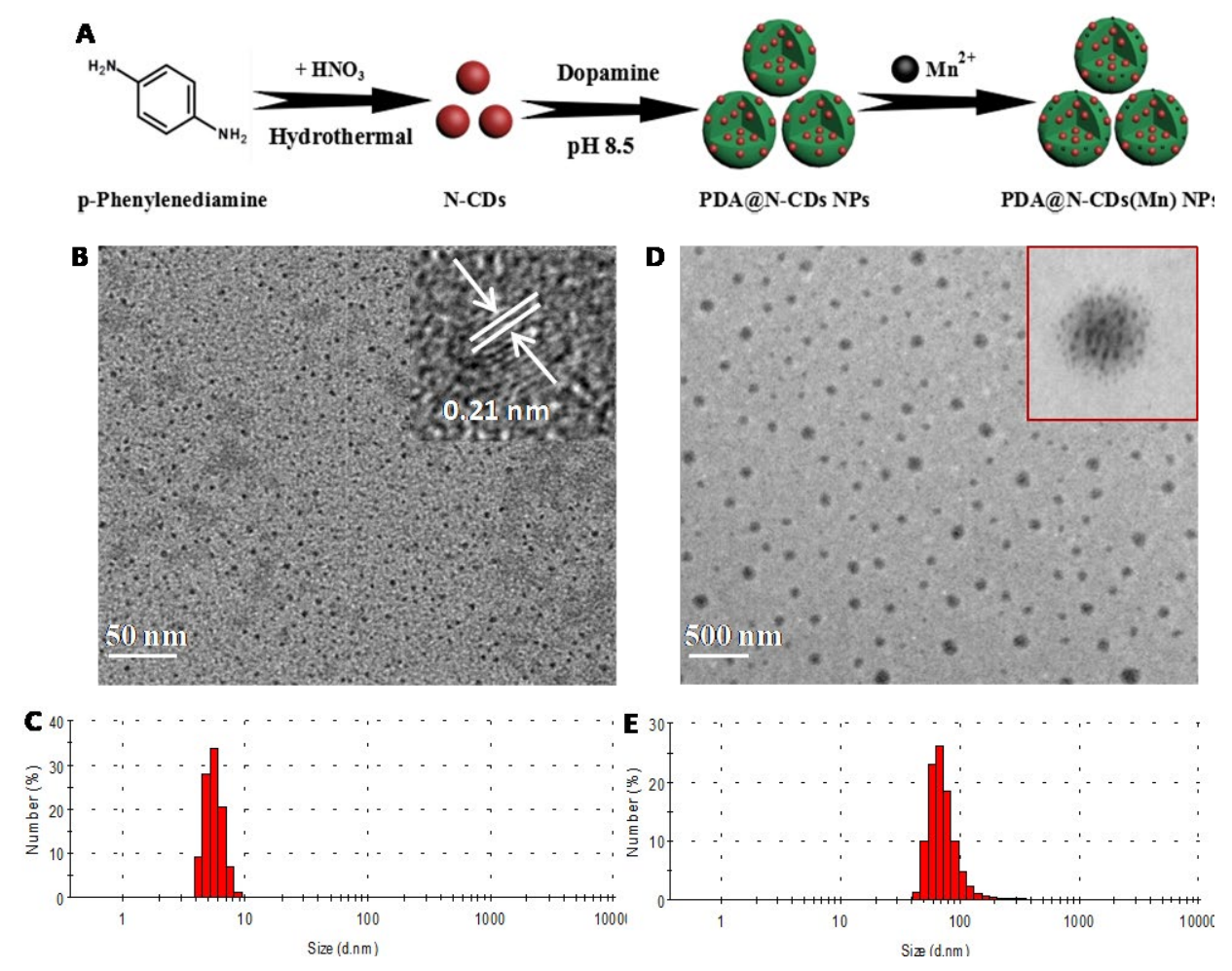

Figure. 1 (A) Synthetic route of PDA@N-CDs(Mn) NPs. (B) TEM image and HRTEM (inset) of N-CDs. (C) DLS measurement of N-CDs. (D) TEM image of PDA@N-CDs(Mn) NPs. Inset: TEM image of single PDA@N-CDs(Mn) NPs. (E) DLS measurement of PDA@N-CDs(Mn) NPs.

The PDA@N-CDs NPs were prepared via a self-polymerization as shown in Fig. 1A [28]. Then, $\mathrm{Mn}^{2+}$ was chelated, acting as MR imaging contrast agent [29]. The phenolic hydroxyl groups of PDA@N-CDs NPs could chelate with $\mathrm{Mn}^{2+}$ at in a mild condition. Fig. 1B showed the transmission electron microscopy (TEM) images of the prepared N-CDs, which indicated that the N-CDs were uniform and monodisperse nanodots. Besides, a crystal lattice notes in the high-resolution TEM (HRTEM) image confirmed their crystalline structure, with labeled interplanar distances of $0.21 \mathrm{~nm}$, which was consistent with the (100) lattice spacing of graphene along the [001] direction (inset in Fig. 1B) [30]. The average diameter of the N-CDs calculated from randomly selected from $100 \mathrm{NPs}$ is $3.2 \mathrm{~nm}$ with uniform size distribution (Fig. S1). Dynamic light scattering (DLS) data shows that N-CDs displayed an average diameter of $5.6 \mathrm{~nm}$ (Fig. 1C), which was slightly larger than that from TEM images. This was because DLS measured the general hydrodynamic size of NPs. TEM images (Fig. S2A) further confirmed the formation of the PDA@N-CDs NPs stabilized by PDA. The resultant PDA@N-CDs(Mn) NPs (Fig. 1B) showed no obvious changes in 
morphology as compared to PDA@N-CDs NPs (Fig. S2A). The PDA@N-CDs(Mn) nanocrystals formed from multiple cores were composed of average $3.3 \mathrm{~nm}$ single $\mathrm{N}$-CDs nanocrystals (inset in Fig. 1D), and the average diameter was $78 \mathrm{~nm}$. It was noticed that N-CDs coated with PDA tended to crowd together on the TEM grid, even though not aggregated among DLS data (Fig. 1E).
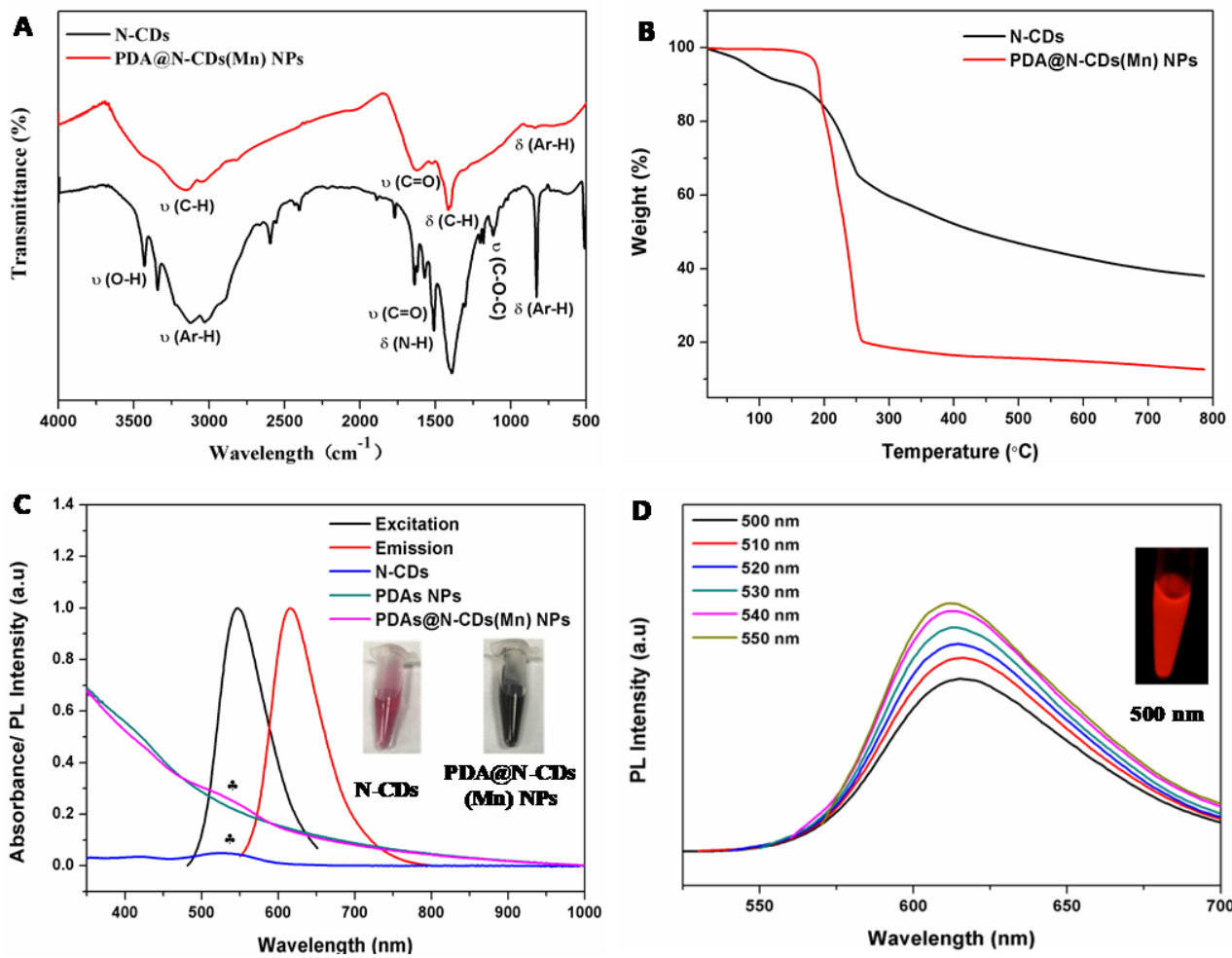

Figure. 2 (A) FT-IR and (B) TGA curves of N-CDs and PDA@N-CDs(Mn) NPs. (C) Normalized UV-vis absorption and fluorescence spectra of PDA@N-CDs(Mn) NPs solution. Insets showed photographs of the PDA@N-CDs(Mn) NPs solution. (D) Normalized emission spectra of PDA@N-CDs(Mn) NPs solution at 500-550 nm excitation wavelengths. The inset showed fluorescence images of PDA@N-CDs(Mn) NPs solution $\left(\lambda_{\mathrm{ex}}=500 \mathrm{~nm}\right)$.

The surface functional groups of the PDA@N-CDs(Mn) NPs were investigated. As shown in Figure 2A, N-CDs possess many groups derived from the precursor, such as $\sigma(\mathrm{N}-\mathrm{H})\left(1520 \mathrm{~cm}^{-1}\right.$, attributing to the deformation vibration of $-\mathrm{NH}_{2}$ or $-\mathrm{NH}$ groups). Other functional groups include $v(\mathrm{O}-\mathrm{H})\left(3500 \mathrm{~cm}^{-1}\right.$, attributing to the deformation vibration of $-\mathrm{OH}$ groups in aliphatic alcohols $), v(\mathrm{C}=\mathrm{O})\left(1600 \mathrm{~cm}^{-1}\right.$, attributing to the existence of $-\mathrm{COOH})$. Finally, the characteristic peak at $1200 \mathrm{~cm}^{-1}$ was attributed to the stretching vibration of $\mathrm{C}-\mathrm{O}-\mathrm{C}$ due to the ring opening and 
oxidation reactions. The FT-IR spectrum of PDA@N-CDs(Mn) NPs showed the typical absorption at $3252.4 \mathrm{~cm}^{-1}$ and $3451.8 \mathrm{~cm}^{-1}$, which came from the bonds of N-H and O-H stretching vibrations, respectively. The absorption peaks at 2952, 2921, and $1378 \mathrm{~cm}^{-1}$ were assigned to the $\mathrm{C}-\mathrm{H}$ stretching and symmetric bending modes of the PDA layer. Moreover, the polymeric structure gave rise to characteristic absorption peaks at 1588 and $1381 \mathrm{~cm}^{-1}$. Figure $2 \mathrm{~B}$ showed the weight loss difference between N-CDs and PDA@N-CDs(Mn) NPs originated from the PDA modification, which indicated that the PDA accounted for 27.4 wt\% in PDA@N-CDs(Mn) NPs. Zeta potential of PDA@N-CDs(Mn) NPs was -22.8 mV (Figure S2B), which suggested that some drugs (ie, doxorubicin, chitosan oligosaccharide, and gefitinib) could be loaded onto the surface of PDA@N-CDs(Mn) NPs through electrostatic interactions [31].

In Figure 2C and Figure 2D, the UV-vis absorption and photoluminescence spectra of the as-synthesized PDA@N-CDs(Mn) NPs was used for investigating its optical properties. The PDA@N-CDs(Mn) NPs solution with the concentration of 250 $\mu \mathrm{g} / \mathrm{mL}$ exhibited a brown color (the inset of Figure 2C), and also had good stability. In terms of the free N-CDs in UV-vis spectra, there was a broad and strong absorption in the NIR region, which was different from the characteristic peaks at $530 \mathrm{~nm}$ and relatively strong absorbance intensities from UV to visible light zone. Notably, the broad and strong NIR absorption made PDA@N-CDs(Mn) NPs became preferred promising photothermal imaging agent for cancer diagnosis with the commonly used NIR laser. The PL spectra of PDA@N-CDs(Mn) NPs in Figure 2D gave clear evidence about the excitation $\left(\lambda_{\mathrm{ex}}\right)$ and emission $\left(\lambda_{\mathrm{em}}\right)$ peaks, corresponding to lines $(550 \mathrm{~nm})$ and blue lines (620 nm), respectively. The photograph of PDA@N-CDs(Mn) NPs dispersion under the excitation wavelength of $500 \mathrm{~nm}$ showed strong red emission (inset of Figure 2D). The surface states which affected the band gap of $\mathrm{N}-\mathrm{CDs}$ were influenced by the $\lambda_{\text {ex }}$-dependent PL behavior $[32,33]$. As a result, investigating the above index was essential for further studying the optical properties of the as-synthesized PDA@N-CDs(Mn) NPs (Figure 2D and Figure S3). The

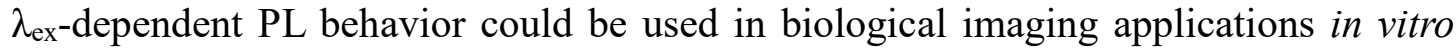


and in vivo [21]. Based on the calculation formula reported by previous literature (Supporting information) [19], the fluorescence quantum yield $\left(\Phi_{\mathbf{S}}\right)$ of the PDA@N-CDs(Mn) NPs was calculated to be 4.5\%.

\subsection{NIR-triggered photothermal effect}
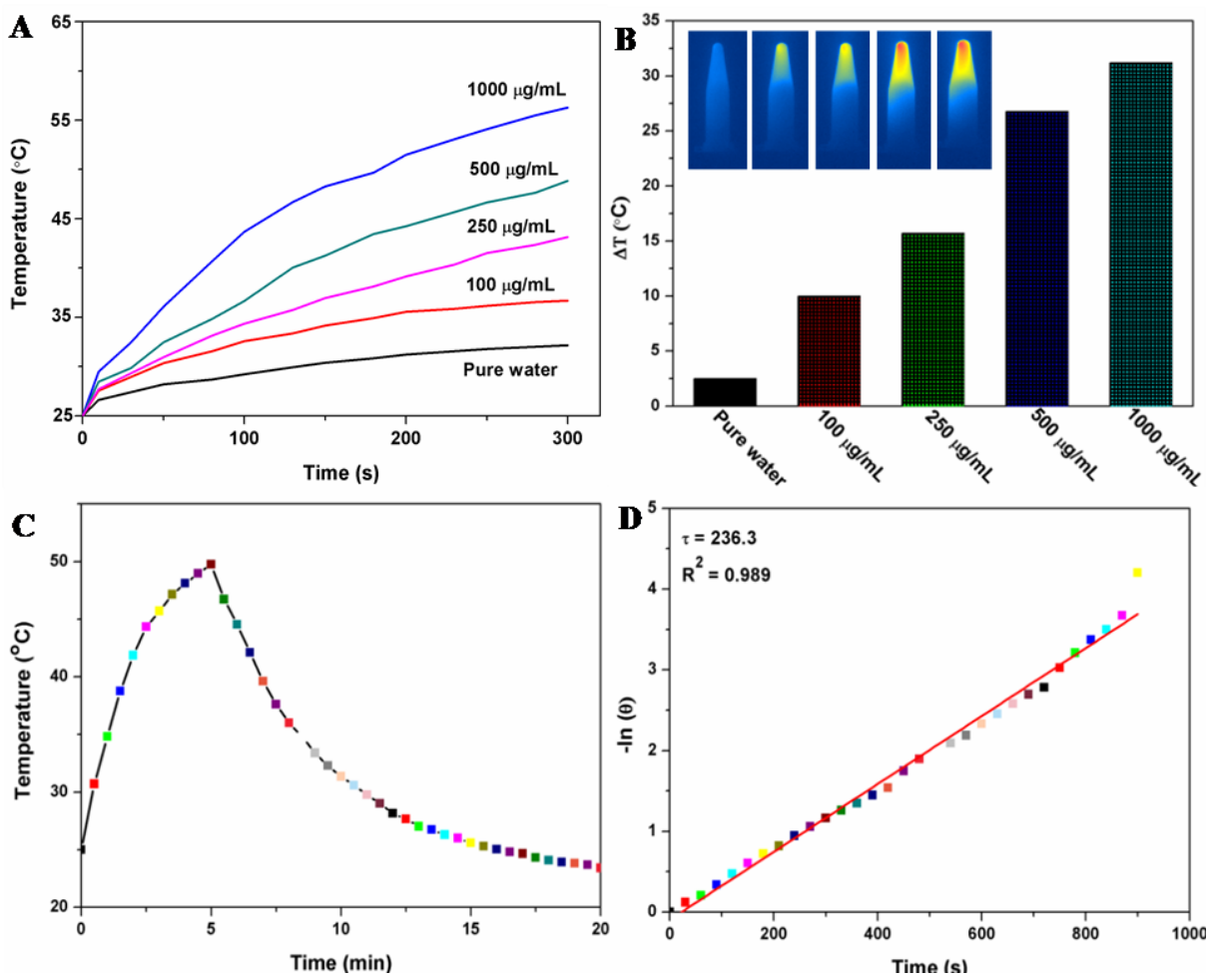

Figure. 3 (A) photothermal responses and (B) changes of pure water and PDA@N-CDs(Mn) NPs solutions with different concentrations exposed with $808 \mathrm{~nm}$ laser $\left(2.0 \mathrm{~W} / \mathrm{cm}^{2}\right)$ for $5 \mathrm{~min}$. (C) Photothermal effect of PDA@N-CDs(Mn) NPs solution exposed with $808 \mathrm{~nm}$ laser $\left(2.0 \mathrm{~W} / \mathrm{cm}^{2}\right)$ for 5 min. (D) Linearity curves fitted from the $-\ln (\theta)$ vs temperature cooling time of PDA@N-CDs(Mn) NPs.

Owing to the extensive absorbance across the whole NIR region of PDA NPs, PDA@N-CDs(Mn) NPs possessed the photothermal effect. PDA@N-CDs(Mn) NPs solutions with ladder-concentrations ( 0 to $1000 \mu \mathrm{g} / \mathrm{mL}$ ) were exposed with an NIR laser $\left(808 \mathrm{~nm}, 2.0 \mathrm{~W} / \mathrm{cm}^{2}\right)$ for $5 \mathrm{~min}$. The results showed that temperatures of the PDA@N-CDs(Mn) NPs solutions increased with the solutions concentrations and irradiation time (Figure 3A and Figure 3B). The temperature of PDA@N-CDs(Mn) NPs solution $(1000 \mu \mathrm{g} / \mathrm{mL})$ increased to about $52.2^{\circ} \mathrm{C}$ (Figure $3 \mathrm{C}$ ), while an increase of only $3.5^{\circ} \mathrm{C}$ was observed in pure water. According to the previous papers that tumor cells would be ablated at $42{ }^{\circ} \mathrm{C}$ for $30 \mathrm{~min}$, whereas the treatment time could be 
extremely shortened by $20-25$ min when the intracellular temperatures exceed $50{ }^{\circ} \mathrm{C}$ [34]. Therefore, the photothermal conversion efficiency $(\eta)$ of PDA@N-CDs(Mn) NPs solution was evaluated to confirm their photothermal capability (Figure 3D). The $\eta$ value of PDA@N-CDs(Mn) NPs was determined to be $28.2 \%$, based on the calculation formula reported [33]. Compared with other PTT materials, such as the $\eta$ value of AuNPs (21\%) [34] and $\mathrm{Cu}_{2-\mathrm{x}} \mathrm{Se}$ NPs (22\%) [35], PDA@N-CDs(Mn) NPs possess a higher $\eta$ value.

\subsection{In vitro biocompatibility of the PDA@N-CDs(Mn) NPs}

Standard MTT assay was performed to test if PDA@N-CDs(Mn) NPs would be an ideal therapeutic agent. Therefore, dilution series of PDA@N-CDs(Mn) NPs were added into wells and incubated with NIH3T3 cells overnight or $24 \mathrm{~h}$. Then, MTT assay was applied to quantify the cell viability. Encouragingly, the data indicated that PDA@N-CDs(Mn) NPs exhibited low dark cytotoxicity even with a high dose and a long incubation time (Figure 4A). Simultaneously, apoptosis and morphology variation as essential indicators were also detected while hardly to observe during the incubated time. Then, biocompatibility was evaluated by living-dead staining, in which cells were distinguished by different colors from Calcein-AM (green fluorescence) to propidium iodide (red fluorescence). In Figure 4B, strong green fluorescence in the whole field showed in groups with different concentrations PDA@N-CDs(Mn) NPs suggested that PDA@N-CDs(Mn) NPs, suggesting possessed good biocompatibility.

As another key factor for its biological application in vivo [36], hemolysis of the as-synthesized material was detected via the hemolytic test. The absorbance of the sample solution to hemoglobin (HGB) was recorded at $540 \mathrm{~nm}$. Figure S4 displayed the hemolytic result and digital photographs of PDA@N-CDs(Mn) NPs solutions with different concentrations. The highest hemolytic efficiency of samples was about $0.14 \%$, which was far below the standard value (5\%). The data supported that the implicit hemolysis effect of the PDA@N-CDs(Mn) NPs could be negligible. 

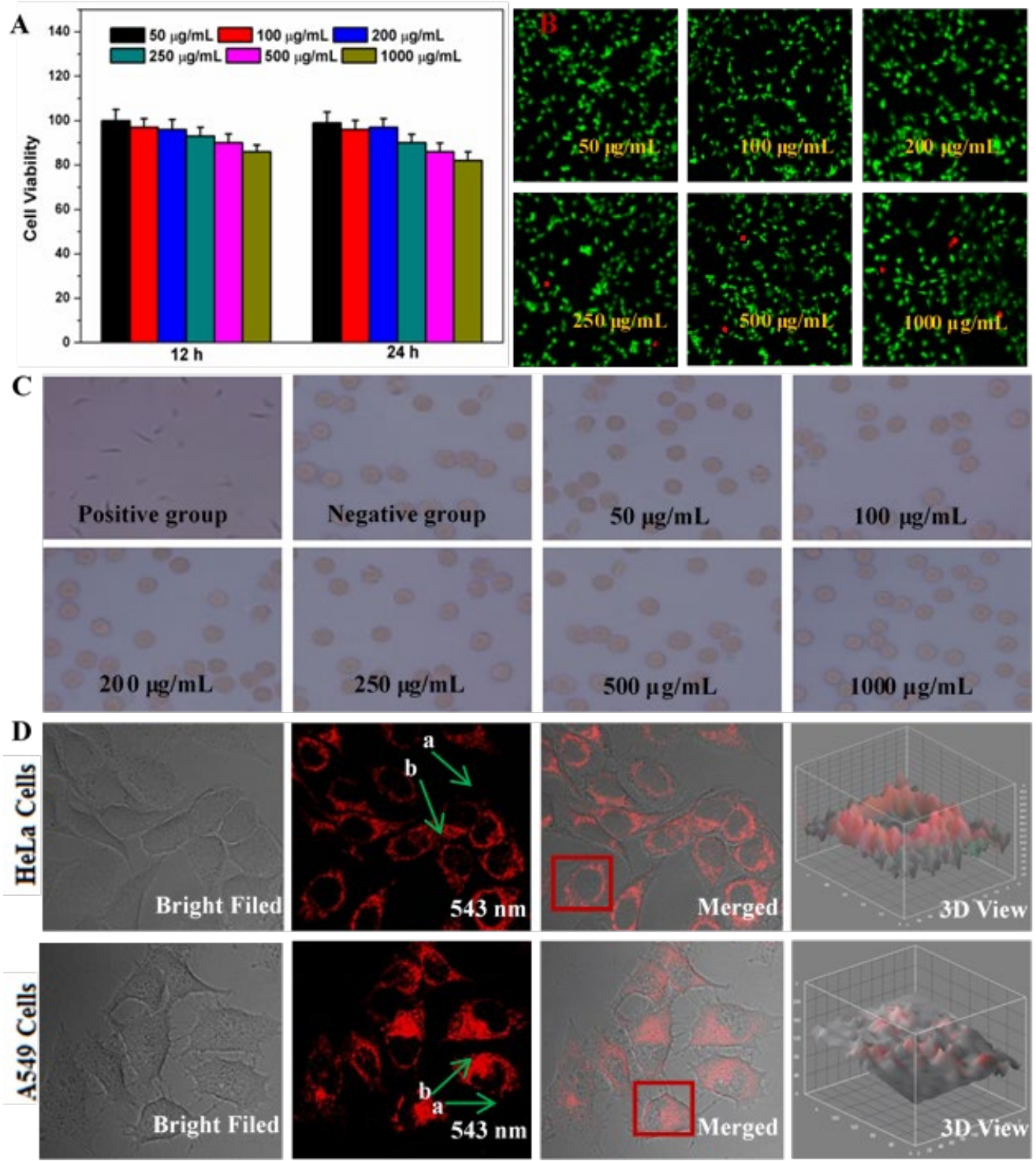

Figure. 4 (A) Viability of NIH3T3 cells treated with PDA@N-CDs(Mn) NPs at different concentrations. (B) Live/dead assays treated at PDA@N-CDs(Mn) NPs at different concentrations. Size bar $=100 \mu \mathrm{m}$ (C) Optical images of RBCs treated with PDA@N-CDs(Mn) NPs at different concentrations. Size bar $=10 \mu \mathrm{m}$ (D) Fluorescent images of HeLa cells and A549 cells treated with PDA@N-CDs(Mn) NPs. (The region of interestwas the area marked with a red rectangle for Z-scan measurement analysis). Size bar $=10 \mu \mathrm{m}$

The crucial point for application of the medical materials in vivo was the interaction between erythrocyte and materials [37]. Aggregation, crenation, and hemolysis acted as indicators of the above interaction. In general, the untreated RBCs in PBS appeared in a common biconcave shape. Figure $4 \mathrm{C}$ showed the general biconcave shape about untreated RBCs in PBS. As reported, materials possessed bad blood compatibility causing the situation of morphological aberrant forms for RBCs 
From incubated RBCs with PDA@N-CDs(Mn) NPs (Figure 4C), it could be concluded that the RBCs could overcome this problem and keep cells with a normal shape at high concentration $(1000 \mu \mathrm{g} / \mathrm{mL})$ of PDA@N-CDs(Mn) NPs. This result agreed with the above hemolysis analysis.

\subsection{In vitro fluorescent imaging of living cells}

The practicability of PDA@N-CDs(Mn) NPs for cells imaging in vitro has been verified using HeLa and A549 cells by CSLM. The fluorescent emission signals were collected at $543 \mathrm{~nm}$, which followed incubation with PDA@N-CDs(Mn) NPs (100 $\mu \mathrm{g} / \mathrm{mL}$ ) at $37{ }^{\circ} \mathrm{C}$ for $30 \mathrm{~min}$. As shown in Figure $4 \mathrm{D}$, fluorescent images and the overlay of the brightfield image indicated that the luminescence came from the intracellular region of cells. Z-scan measurements further supported the fact that PDA@N-CDs(Mn) NPs had successfully entered into the cells. Besides, a clear fluorescent emission was collected from the transversal section in the region of interest. Finally, based on the above results, which indicated that the PDA@N-CDs(Mn) NPs had been internalized into the cells. The load rate of PDA@N-CDs(Mn) NPs in living cells was calculated by a quantitative analysis based on the intensity of the fluorescent signal and the background. Fluorescent intensity distribution inside HeLa cells was labeled with a red rectangle in Figure S5. The fluorescent signals of background noise (point a) were close to 2, whereas the calculated green fluorescent intensity (point b) was more than 54. A similar fluorescent intensity over 61 in A549 cells was detected (Figure S5). Such high fluorescent intensity strongly suggested that PDA@N-CDs(Mn) NPs could serve as a potential probe for bioimaging.

\subsection{In vivo photothermal imaging}

Inspired by the high photothermal conversion efficiency of PDA@N-CDs(Mn) NPs, in vivo photothermal imaging with PDA@N-CDs(Mn) NPs was performed. The tumor-bearing mice were intravenously injected with $100 \mu \mathrm{L}$ aqueous dispersion of PDA@N-CDs(Mn) NPs $(1000 \mu \mathrm{g} / \mathrm{mL})$ or PBS, and then exposed to the NIR laser 
$\left(808 \mathrm{~nm}, 2.0 \mathrm{~W} / \mathrm{cm}^{2}\right)$ for $5 \mathrm{~min}$. In PBS group, the final temperature showed a negligible increase of less than $1.5^{\circ} \mathrm{C}$ within 5 min irradiation (Figure $5 \mathrm{~A}$ and Figure 5B). In contrast, the tumor temperature of PDA@N-CDs(Mn) NPs group rapidly increased from $33.5{ }^{\circ} \mathrm{C}$ to $50.6{ }^{\circ} \mathrm{C}$ within $5 \mathrm{~min}$ (Figure S6). The photothermal imaging showed that PDA@N-CDs(Mn) NPs were preferentially accumulate in the tumor, which was mainly due to the enhanced permeability and retention (EPR) effect.

\subsection{In vivo MR imaging}

Before in vivo MR imaging, the metabolic behavior of PDA@N-CDs(Mn) NPs were examined. The injection of PDA@N-CDs(Mn) NPs in female Balb/c mice via the vein. Blood of mice was collected at every time point and further dissolved with lysis buffer. Then, the blood was extracted with $\mathrm{HCl} /$ isopropanol to determine concentrations of PDA@N-CDs(Mn) NPs through the fluorescence signal. As shown in Figure S7, the PDA@N-CDs(Mn) NPs signals gradually reduced over time following a two-compartment model, with the first $\left(\mathrm{t}_{1 / 2}(\alpha)\right)$ and second $\left(\mathrm{t}_{1 / 2}(\beta)\right)$ phases of circulation half-lives determined to be $0.49 \pm 0.15 \mathrm{~h}$ and $6.32 \pm 0.49 \mathrm{~h}$, respectively.

Interestingly, the prepared PDA@N-CDs(Mn) NPs featured as a brightening effect with concentration variation manner under $\mathrm{T}_{1}$-weighted MR imaging (Figure 5C). In contrast, $\mathrm{T}_{2}$-weighted $\mathrm{MR}$ imaging showed a darkening effect (Figure 5D). The $\mathrm{T}_{1}$ relaxivity $\left(\mathrm{r}_{1}\right)$ of PDA@N-CDs(Mn) NPs were measured to be $14.15 \mathrm{mM}^{-1} \mathrm{~s}-1$ and $\mathrm{T}_{2}$ relaxivity $\left(\mathrm{r}_{2}\right) 39.2 \mathrm{mM}^{-1} \mathrm{~s}^{-1}$, respectively. Thus, Mn-doped PDA@N-CDs(Mn) NPs could be used for $\mathrm{T}_{1}$-weighted or $\mathrm{T}_{2}$-weighted $\mathrm{MR}$ imaging. 

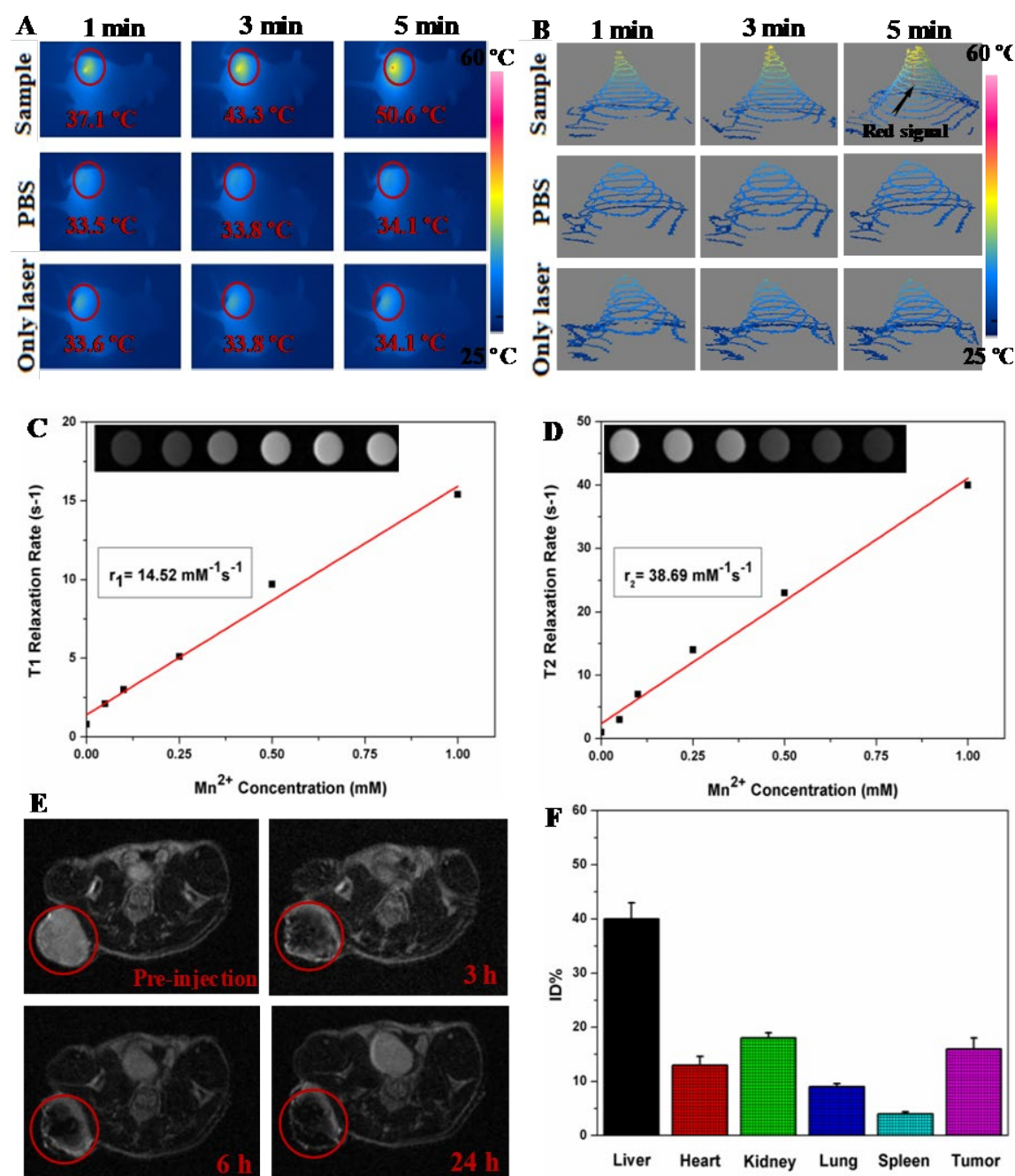

Figure. 5 (A) The infrared thermal camera images of the HeLa tumor-bearing mice injected with PDA@N-CDs(Mn) NPs and PBS exposed with NIR laser irradiation. (B) The 3D infrared thermal camera images of the tumor region of (A). (C-D) T1 and T2 relaxation rates of PDA@N-CDs(Mn) NPs solutions at different $\mathrm{Mn}^{2+}$ concentrations. (E) T2-weighted MR images of HeLa tumor-bearing mice taken before injection and 1, 3, 6, and $24 \mathrm{~h}$ post injection of PDA@N-CDs(Mn) NPs. (F) Biodistribution of PDA@N-CDs(Mn) NPs in mice.

In vivo experiments were performed to investigate the targeting efficiency of PDA@N-CDs(Mn) NPs. The intravenous injection of PDA@N-CDs(Mn) NPs in A549 tumor-bearing mice and then imaged under an MR imaging system. After $24 \mathrm{~h}$, a remarkable darkening effect could be found in the tumor tissue (Figure 5E). Moreover, the ROI quantification of MR imaging also indicated the increase of $\mathrm{T}_{2}$-weighted MR signals, which further proved the effective accumulation of NPs in the tumor (Figure S8). Then, inductively coupled plasma-atomic emission spectrometry (ICP-AES) showed clear identification that accumulation of large 
amounts $\mathrm{Mn}^{2+}$ in the reticuloendothelial (liver) system and urinary (kidney) system at $24 \mathrm{~h}$ post-injection (Figure 5F). Particularly, the $\mathrm{Mn}^{2+}$ content of the tumor could achieve $21.5 \%$ of the injected dose, which indicated a high targeting efficacy of the PDA@N-CDs(Mn) NPs.

\subsection{In vivo fluorescence imaging}
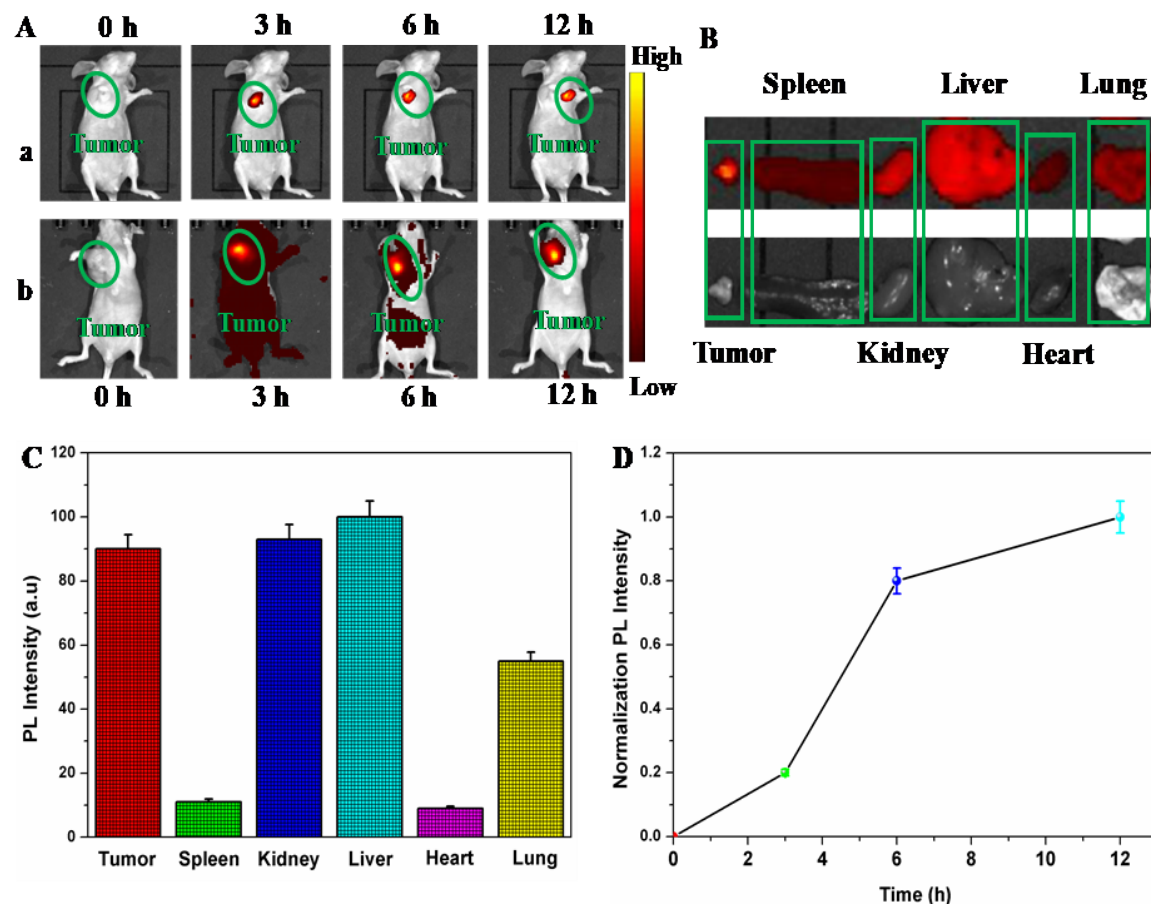

Figure 6. (A) Real-time in vivo red fluorescence images obtained after (a) subcutaneous and (b) intravenous injections of PDA@N-CDs(Mn) NPs in nude mice via the tail vein at different time points. (B) Ex vivo images of mice tissues. (C) Biodistribution of PDA@N-CDs(Mn) NPs in major organs excised from mice $12 \mathrm{~h}$ post-intravenous injection. (D) Average fluorescence intensities from the tumor area $12 \mathrm{~h}$ post-intravenous injection.

In order to explore in vivo fluorescence with a Maestro 2 Multispectral Small-Animal Imaging System, $20 \mu \mathrm{L}$ of PDA@N-CDs(Mn) NPs dispersed in PBS was injected onto the left rear flank region of the animal model. The subcutaneous injection site displayed a strong fluorescence intensity (Figure 6A, a). The region of the fluorescence intensity gradually expanded from the center that suggested that PDA@N-CDs(Mn) NPs had good distribution ability in tissues. In order to further study the possible application of PDA@N-CDs(Mn) NPs for in vivo theranostics, fluorescence imaging was firstly used to investigate the A549 tumor-bearing nude 
mice treated with or without intravenous injection of PDA@N-CDs(Mn) NPs. Figure 6A, b revealed that the PDA@N-CDs(Mn) NPs accumulated in the tumor tissue via the EPR effect and valid fluorescence could be seen around the tumor [38]. After $12 \mathrm{~h}$ post-injection, mice were sacrificed and then primary organs (i.e., heart, liver, spleen, lung, and kidney) and tumors were harvested which were imaged to assess fluorescence intensities (Figure 6B).PDA@N-CDs(Mn) NPs mainly accumulated in the liver, lung, and kidney tissues (Figure 6C), which was similar to the previous research carbon nanosphere [39]. Stronger fluorescence signal could be found in tumor tissues than the spleen and heart by the time-dependent fluorescence intensity manner (Figure 6D). Thus, these results indicate that green and red-emissive PDA@N-CDs(Mn) NPs would be a promising fluorescence imaging agent.

\subsection{In vivo biocompatibility of the PDA@N-CDs(Mn) NPs}
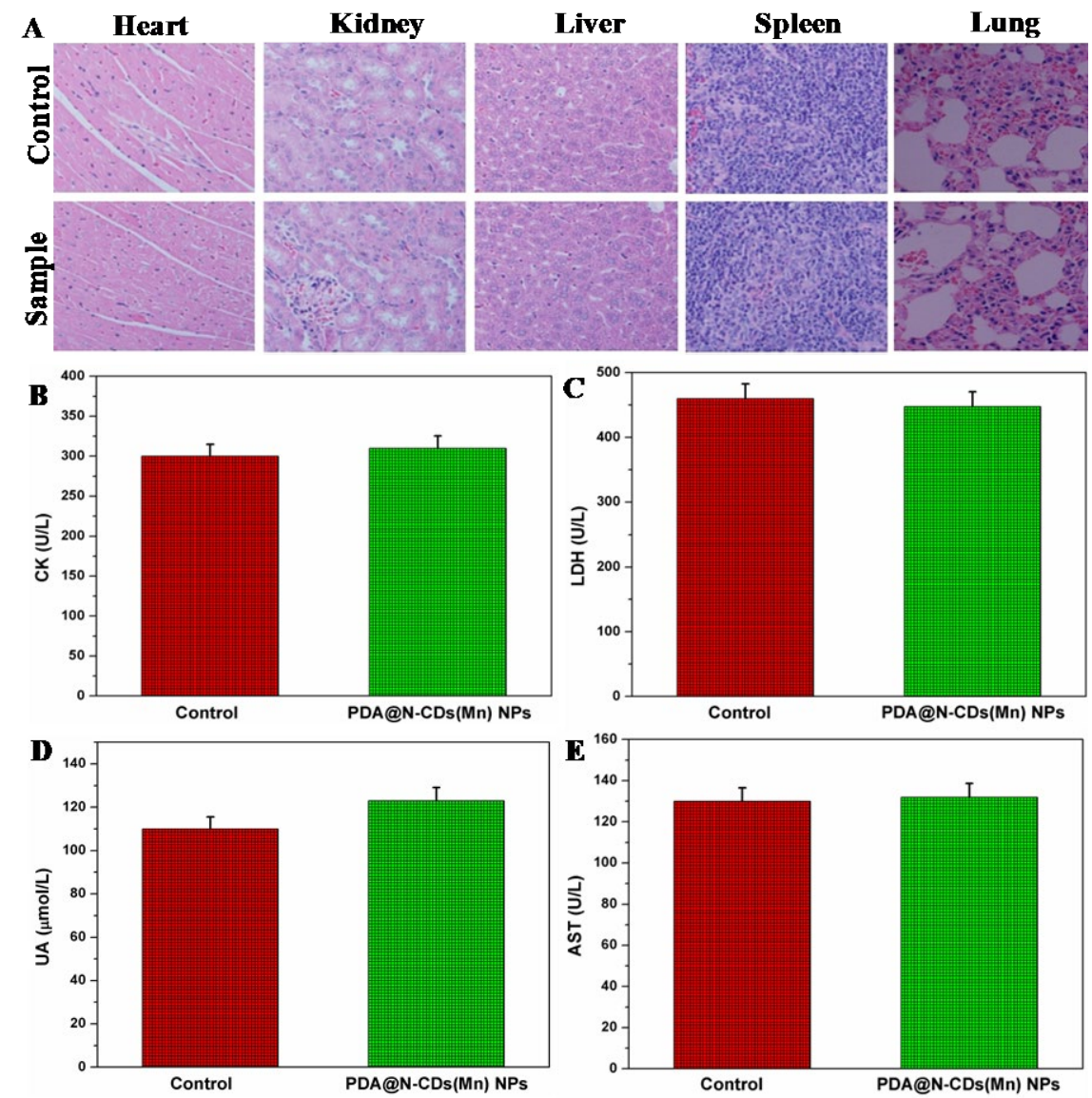

Figure 7. (A) H\&E staining of main organs sections collected from various mice groups after 21 days. (B-E) Blood biochemistry analysis of the mice treated with PDA@N-CDs(Mn) NPs after 21 
days.

In this work, the biosafety of PDA@N-CDs(Mn) NPs in mice was detected through H\&E staining (Figure 7A) after injecting for 21 days. As a result, all mice kept common behavior and symptoms, the normality stayed the same in mice organs and the normal tissues. Moreover, the toxicity of PDA@N-CDs(Mn) NPs was evaluated through blood biochemical analysis. The Creatine Kinase (CK) and Lactate Dehydrogenase $(\mathrm{LDH})$ severed as critical parameters to test the toxicity of heart function, which indicated a negligible acute of the PDA@N-CDs(Mn) NPs (Figure 7B-E). In addition, the parameters analysis of kidney function [Uric Acid (UA)] and liver function [Aspartate Aminotransferase (AST)] showed no damage to the kidney and liver. Above all, it could be summarized that PDA@N-CDs(Mn) NPs had good biocompatibility. All results illustrate that PDA@N-CDs(Mn) NPs is a promising nanocomposite for targeting tumor imaging.

\section{Conclusion}

In summary, novel multifunctional PDA@N-CDs(Mn) NPs that exhibited green and red dual emission, high stability, and excellent water solubility have been successfully synthesized. Because of the high mass extinction coefficient at a NIR laser irradiation, PDA@N-CDs(Mn) NPs exhibited excellent photothermal effects with a high photothermal conversion efficiency of 28.2\%. Notably, PDA@N-CDs(Mn) NPs showed high contrast and spatial resolution of photothermal, fluorescent, and MR imaging efficiency, as well as excellent targeting accumulation at tumor sites. Moreover, histological analyses and blood biochemical analysis verified that PDA@N-CDs(Mn) NPs do not lead to obvious toxicological responses. This work suggests that PDA@N-CDs(Mn) NPs can be used as active agents in photothermal, fluorescent and MR imaging, which significantly expands their applications in like bioimaging.

\section{Acknowledgement}


This work was supported by the Jiangsu six category outstanding talent (2012-NY-031), Jiangsu province science and technology support plan (BE2015367), and the Villum Fonden, Denmark, Project No. 13153. B.S., M.Z., and T.Z. would like to thank the CSC for its generous support.

\section{References}

[1] A. Forsberg, H. Engler, O. Almkvist, G. Blomquist, G, Hagman, A. Wall, Neurobiol Aging 29 (2008) 1456-1465.

[2] C. D. Johnson, M.H. Chen, A.Y. Toledano, J. P. Heiken, A. Dachman, M. D. Kuo, New Engl. J. Med. 359 (2008) 1207-1217.

[3] J. Zhou, Z. Liu, F. Y. Li, Chem. Soc. Rev. 41 (2012) 1323-1349.

[4] J. Ge, Q. Jia, W. Liu, M. Lan, B. Zhou, L. Guo, H.Y. Zhou. H.Y. Zhang, Y. Wang,Y. Gu, X.M. Meng, P.F. Wang, Adv. Healthcare Mater. 5 (2016) 665-675.

[5] O. Akhavan, G. Elham, E. Hamed, J. Mater. Chem. 22 (2012): 20626-20633.

[6] M. Zhang, W. Wang, N.L. Zhou, P. Yuan, Y.T. Su, M.N. Shao, C. Chi, P.Y. Pan, Carbon, 118 (2017) 752-764.

[7] X.Y. Liang, W.T. Shang, C.W. Chi, C.T. Zeng, K. Wang, C.H. Fang, Q.S. Chen, H.Y. Liu., H.F. Fan, J. Tian, Cancer Lett. 383 (2016) 243-249.

[8] W. Tao, X.B. Zhu, X. Yu, X.W. Zeng., H. Zhang. H. Mei., Adv. Mater. 29 (2017) 1603276.

[9] L. Cheng, J.J. Liu, X. Gu, H. Gong, Z. Liu., Adv. Mater. 26(2014) 1886-1893.

[10] M. Liu, X. Zhang, B. Yang, Z. Li, F. Deng, Y. Yang, X. Zhang, Y. Wei, Carbohydr. Polym. 121 (2015) 49-55.

[11] C. Liu, P. Zhang, X. Zhai, F. Tian, W. Li, J. Yang, Y. Liu, H. Wang, W. Wang, W. Liu, Biomaterials. 33 (2012) 3604-3613.

[12] L. Cao, X. Wang, M.J. Meziani, F. Lu, H. Wang, P. G. Luo, Y. Lin, B. A. Harruff, L. M. Veca, D. Murray, S. Y. Xie, Y. P. Sun, J. Am. Chem. Soc. 129 (2007) 11318-11319.

[13] H. Yu, R. Shi, Y. Zhao, G. I. N. Waterhouse, L. Z. Wu, C. H. Tung, T. Zhang, Adv. Mater. 28 (2016) 9454-9477.

[14] J. C. Ge, Q. Y. Jia, W. M. Liu, L. Guo, Q. Y. Liu, M. H. Lan, H. Y. Zhang, X. M. Meng, P. F. Wang, Adv. Mater. 27 (2015) 4169-4177.

[15] L. Bao, C. Liu, Z. L. Zhang, D. W. Pang, Adv. Mater. 27 (2015) 1663-1667.

[16] M. Vedamalai, A. P. Periasamy, C. W. Wang, Y. T. Tseng, L. C. Ho, C. C. Shih, H. T. Chang, Nanoscale. 6 (2014) 13119-13125.

[17] T. S. Xu, X. Y. Li, Z. H. Xie, X. G. Li, H. Y. Zhang, Microchim. Acta. 182 (2015) 2541-2549.

[18] X. Gong, Q. Zhang, Y. Gao, S. Shuang, M. M. Choi, C. Dong, Appl. Mater. Interfaces 8 (2016) 11288-11297.

[19] M. Zhang, W. Wang, Y. Cui, N. Zhou, J. Shen, ACS Biomater. Sci. Eng. 4 (2018) 151-162

[20] Z. Wang, H. Liao, H. Wu, B. Wang, H. Zhao, M. Tan, Anal. Methods 7 (2015) 8911-8917.

[21] M. Zhang, C. Chi, P. Yuan, Y. Su, M. Shao, N. Zhou, Mater. Sci. Eng. C 76 (2017) $1146-1153$.

[22] Z. Dong, H. Gong, M. Gao, W. Zhu, X. Sun, L. Feng, Theranostics. 6 (2016) 1031-1042. 
[23] Y. Liu, K. Ai, L. Lu, Chem Rev. 114 (2014) 5057-5115.

[24] B. Liu, C. Li, B. Xing, J Lin, J. Mater. Chem. B. 4 (2016), 4884-4894.

[25] Y. Liu, K. Ai, J. Liu, M. Deng, Y. He, L. Lu, Adv Mater. 25 (2013) 1353-1359.

[26] Y.Y. Li, C.H. Jiang, D.W. Zhang, Y. Wang, X.Y. Ren, K.L. Ai, X.S. Chen, L.L. Lu, Acta Biomater. 47 (2017) 124-134.

[27] J.D. Shao, H.H. Xie, H. Huang, Z.B. Li, Z.B. Sun, Y.H. Xu, Q.L. Xiao, H.F. Yu, Y.T. Zhao, H. Zhang H.Y. Wang, P.K. Chu, Nat. Commun. 7 (2016) 12967.

[28] J. Xi, L. Da, C. Yang, R. Chen, L. Gao, L. Fan, J. Han, Int. J. Nanomed. 12 (2017) $3331-3345$.

[29] L. S. Lin, Z. X. Cong, J. B. Cao, K. M. Ke, Q. L. Peng, J.H. Gao, H.H. Yang, G. Liu, H.Y. Chen, ACS Nano. 8 (2014) 3876-3883.

[30] T. N. J. I. Edison, R. Atchudan, J. J. Shim, S. Kalimuthu, B. C. Ahn, Y. R. Lee, J. Photochem. Photobiol., B 158 (2016) 235-242.

[31] H. Ding, F. Du, P. Liu, Z. Chen, J. Shen, ACS Appl. Mater. Interfaces 7 (2015) 6889-6897.

[32] S. Zhuo, M. Shao, S. T. Lee, ACS Nano 6 (2016) 1059-1064.

[33] J. Shang, L. Ma, J. Li, W. Ai, T. Yu, G. G. Gurzadyan, Sci. Rep. 2 (2012) 00792.

[34] H. L. Chen, Z. M. Liu, S. Y. Li, H. Q. Zhong, Z. Y. Guo, Theranostics, 6 (2016) 1096-1104.

[35] S. H. Zhang, C. X. Sun, J. Zeng, M. Y. Gao, Z. Li, Adv. Mater. 28 (2016) 8927-8936.

[36] W. Li, P. Rong, K. Yang, P. Huang, K. Sun, X. Chen, Biomaterials, 45 (2015) 18-26.

[37] J. Tan, N. Meng, Y. Fan, Y. Su, M. Zhang, Y. Xiao, N. Zhou, Mater. Sci. Eng. C 61 (2016) 681-687.

[38] R. Duncan, L. Izzo, Adv. Drug Delivery Rev. 57 (2015) 2215-2237.

[39] S. Q. Chen, Q. Y. Jia, X. L. Zheng, Y. M. Wen, W. M. Liu, H. Y. Zhang, J. C. Ge, P. F. Wang, Sci. China Mater. 61 (2018) 1325-1338. 